\title{
Consumo alimentar, estado nutricional e nível de atividade física em comunidade universitária brasileira ${ }^{1}$
}

\author{
Food intake, nutritional status and level of \\ physical activity in a Brazilian \\ university community
}

Erika da Silva MACIEL ${ }^{2}$

Jaqueline Girnos SONATI

Denis Marcelo MODENEZE ${ }^{3}$

Julia Santos VASCONCELOS²

Roberto VILARTA ${ }^{3}$

\section{R E S U M O}

\section{Objetivo}

Este estudo teve como objetivo avaliar a variabilidade e a frequência do consumo alimentar, o estado nutricional e o nível de atividade física em uma comunidade universitária brasileira.

\section{Métodos}

A amostra constitui-se de 303 voluntários (130 homens e 173 mulheres) pertencentes à comunidade universitária do interior de São Paulo e avaliada por meio da disponibilização pela Internet do Questionário de Frequência Alimentar do International Physical Activity Questionaire e por meio da coleta da descrição autorreferida do peso e da altura. Foi realizada análise descritiva dos dados, análise de frequência alimentar e teste do coeficiente de correlação de Spearman.

\section{Resultados}

Foram observados inadequação dos hábitos alimentares, sobrepeso e obesidade na amostra estudada. As correlações entre os grupos alimentares apresentaram valores de magnitude maiores no grupo de mulheres que se alimentam de forma mais adequada do que os homens, mas não foram observadas diferenças entre os

1 Artigo elaborado a partir da dissertação de ES MACIEL, intitulada "Qualidade de vida: análise da influência do consumo de alimentos e estilo de vida". Universidade de São Paulo; 2006

2 Universidade de São Paulo, Escola Superior de Agricultura "Luiz de Queiroz", Centro de Energia Nuclear na Agricultura. Av. Pádua Dias, 11, 13418-260, Piracicaba, SP, Brasil. Correspondência para/Correspondence to: ES MACIEL. E-mail: <erikasmaciel@gmail.com>.

${ }^{3}$ Universidade Estadual de Campinas, Faculdade de Educação Física. Campinas, SP, Brasil. 
708 ES MACIEL et al.

sexos no nível de atividade física. Não houve correlação significativa entre grupos de alimentos consumidos, índice de massa corporal e nível de atividade física em ambos os sexos.

\section{Conclusão}

A comunidade universitária estudada apresentou baixo consumo de frutas, legumes e verduras, bem como consumo insuficiente de alimentos do grupo de cereais e leguminosas.

Termos de indexação: Atividade física. Consumo alimentar. Estado nutricional.

\section{A B S T R A C T}

\section{Objective}

This study assessed the food intake variability and frequency, nutritional status and level of physical activity of a university community in Brazil.

\section{Methods}

The sample comprised 303 volunteers (130 men and 173 women) from a university community in São Paulo state. They answered the International Physical Activity Questionnaire online and reported their weight and height. Statistical treatment included descriptive analysis, analysis of food intake frequency (Dietsys) and Spearman's rank correlation coefficient.

\section{Results}

Eating habits were inappropriate and many individuals were overweight or obese. There were more correlations among food groups for women who had better diets than men but the level of physical activity did not differ between the genders. There was no significant correlation between food groups and body mass index or level of physical activity for men or women.

\section{Conclusion}

The study university community consumes little fruit, non-starchy vegetables, grains and legumes.

Indexing terms: Physical activity. Food consumption. Nutritional status.

\section{N T R O D U ÇÃ O}

É crescente a divulgação nas mídias de uma possível relação entre as escolhas cotidianas de consumo alimentar e a perspectiva de melhoria da condição nutricional individual relativa ao peso corporal. Essa visão está geralmente associada a recomendações para a adoção de estilo de vida saudável, estruturado por práticas corporais dirigidas à otimização do gasto energético e à manutenção das capacidades e habilidades vitais.

As escolhas cotidianas de consumo alimentar realizadas pela população leiga, de modo geral, são guiadas pela influência de fatores que perpassam os aspectos das condições de vida mais gerais, como nível de renda, urbanização local e acesso à variabilidade de alimentos, incluindo também as características individualizadas de nível educacional, faixa etária e cultura alimentar familiar.
A abordagem científica relacionada ao consumo de alimentos confirma o senso comum ao demonstrar existir uma correlação entre as condições de vida e as opções pessoais de adoção de estilos de vida, além da influência de fatores sensoriais, socioeconômicos e de disponibilidade dos alimentos, o que, no entanto, não assegura que as escolhas alimentares sejam necessariamente saudáveis'.

Hábitos alimentares saudáveis fazem parte de estratégias para promoção da saúde, aspecto sustentado por estudos epidemiológicos que, durante pelo menos duas décadas, relacionaram dietas ricas em frutas, vegetais e cereais à menor incidência de doenças crônicas não transmissíveis e à melhor qualidade de vida ${ }^{2,3}$.

Tais constatações têm gerado pesquisas que visam a compreender a relação entre dieta e saúde a partir da análise das frequências de consu- 
mo de alimentos e do estado nutricional diante dos aspectos determinantes de doenças e indicadores de morbidade e mortalidade ${ }^{4,3}$.

A Organização Mundial da Saúde (OMS) 5 recomenda a ingestão diária de pelo menos cinco porções, ou, aproximadamente, 400 gramas, de frutas e verduras. Já no Brasil, o Ministério da Saúde recomenda o consumo diário de três porções de frutas e três de legumes e verduras, informações disponibilizadas no Guia Alimentar para População Brasileira6 .

No entanto, mesmo em países europeus que detêm tradição de maior consumo de frutas, legumes e verduras, observa-se tendência de transição nutricional, indicando aumento da dieta rica em gorduras saturadas e açúcares e reduzida em carboidratos complexos, leguminosas, pescado, frutas e verduras, principalmente nas gerações mais novas, formadas por crianças e jovens².

Dados recentes indicam que, no Brasil, há baixo consumo de frutas, legumes e verduras na maioria das faixas etárias da população, relacionado à maior morbidade, em especial de doenças crônicas não transmissíveis e excesso de peso corporal $^{7-11}$. Aliado a esse fator, há o declínio progressivo da atividade física diária, cuja associação com dietas inadequadas pode determinar alterações indesejáveis na composição corporal ${ }^{10}$.

A adoção de dietas saudáveis pode ter relação com a prática de atividade física, visto haver relação entre estado nutricional e atividade física vinculada à execução de tarefas da vida diária e de práticas de caminhadas, esporte e lazer ativo $^{12,13}$.

Diante desses dados e considerando a importância da ingestão diária de frutas, legumes e verduras como estratégia de promoção da saúde, este estudo teve como objetivo avaliar a variabilidade e a frequência do consumo alimentar, estado nutricional e nível de atividade física, bem como verificar possíveis associações entre essas variáveis em comunidade universitária, buscando uma melhor compreensão do tema.

\section{MÉ T O D O S}

O estudo utilizou um corte transversal exploratório, com amostragem não probabilística de 303 voluntários pela estratégia de conveniência de forma consecutiva de uma população-alvo do Campus da Escola Superior de Agricultura "Luiz de Queiroz" (ESALQ) da Universidade de São Paulo, constituída por uma comunidade de aproximadamente 3752 pessoas: 1750 alunos de graduação, 951 alunos de pós-graduação, 222 funcionários docentes e 834 funcionários não docentes. O critério de inclusão foi possuir um webmail do sistema ESALQ/USP e vínculo com o campus nas condições de aluno ou servidor.

O projeto de pesquisa foi aprovado pelo Comitê de Ética em Pesquisa da Faculdade de Odontologia de Piracicaba (Protocolo CEP/FOP/ UNICAMP n 143/2004), e todos os participantes concordaram em participar e assinar o Termo de Consentimento Livre e Esclarecido antes de sua inclusão na amostra. A coleta de dados foi realizada entre setembro de 2005 e março de 2006, e o tratamento dos dados entre novembro de 2006 e junho de 2007, sendo apresentadas devolutivas das principais informações à comunidade universitária entre setembro de 2008 e maio de 2009. Todas as ações educativas sobre os dados coletados e os principais resultados nessa comunidade universitária fizeram parte de um amplo conjunto de ações inseridas em programas de promoção da saúde nos campi da USP, visando a um maior conhecimento dos hábitos das comunidades universitárias.

\section{Instrumentos}

A análise da frequência alimentar privilegiou o foco sobre "grupos de alimentos", visando à identificação da frequência de consumo de alimentos e/ou grupo de alimentos no período de 15 dias, e considerou o consumo usual dos alimentos pré-determinados pelo pesquisador, favorecendo maior objetividade e baixo custo operacional ${ }^{14}$. 
A seleção dos alimentos que integram o formulário de frequência alimentar foi baseada na literatura disponível, especificamente no que diz respeito às substâncias presentes em tais alimentos (alimentos funcionais) e seus reconhecidos benefícios para a saúde humana. As porções foram padronizadas, sendo mantidas com valores fixos durante toda a fase de coleta de dados, e os alimentos classificados em grupos de bebidas, cereais, verduras, óleos e temperos, leguminosas e frutas.

No grupo de bebidas, foram consideradas as constituídas de nutrientes funcionais e consumidas pelos voluntários, como chá verde (função antioxidante), sucos naturais (antioxidantes e fonte de vitaminas), suco de uva (anticarcino-gênico e antioxidante), bebidas à base de soja, leite (fonte de cálcio), bebidas fermentadas (probióticos e prébioticos), bebidas estimulantes, como o café, além das bebidas alcoólicas, como vinho tinto e cerveja ${ }^{15-17}$.

Os outros grupos foram inseridos a fim de se verificar o consumo de alimentos ricos em fibras (cereais e verduras), ômega-6 e gorduras saturadas (óleos e temperos), proteína vegetal (leguminosas) e, por último, o grupo das frutas (carboidratos, vitaminas e sais minerais) (Quadro 1).

A amostra foi ainda classificada quanto ao Nível de Atividade Física (NAF) pelo International Physical Activity Questionaire (IPAQ), versão curta e semana normal, desenvolvido pela OMS, vali- dado, avaliado e utilizado no Brasil e em outros países ${ }^{18-20}$, contendo oito perguntas em relação à frequência e à duração da realização de atividades físicas moderadas, vigorosas e de caminhada, tendo como referência a última semana ou uma semana típica.

As atividades físicas vigorosas são aquelas que demandam um grande esforço físico e fazem com que o indivíduo respire muito mais forte do que o normal, enquanto as atividades físicas moderadas são aquelas que exigem algum esforço físico e fazem com que o indivíduo respire um pouco mais forte que o normal ${ }^{21}$.

A versão utilizada neste estudo apresenta bons coeficientes de validade e reprodutibilidade, com a vantagem da forma curta ser prática, rápida e possibilitar levantamento de dados junto a grandes grupos populacionais. A forma curta é mais aceita pelos participantes, pelo fato de a forma longa ser considerada repetitiva e cansativa para a emissão das respostas pelos entrevistados ${ }^{22}$. Os dados permitem classificar os sujeitos quanto ao nível de atividade física nas seguintes categorias:

Sedentário: aquele indivíduo que não realizou atividade física durante pelo menos dez minutos contínuos ao longo da semana.

Insuficientemente ativo: aquele indivíduo que realiza atividade física com duração mínima de dez minutos por semana, porém insuficiente para ser classificado como ativo. Esses indivíduos ainda podem ser classificados em dois grupos, a saber:

Quadro 1. Descrição dos grupos de alimentos que compuseram o questionário de frequência alimentar dos voluntários da comunidade do Campus "Luiz de Queiroz". Piracicaba (SP), 2006.

\begin{tabular}{ll}
\hline Grupo de alimentos & Integrantes \\
\hline Bebidas & $\begin{array}{l}\text { Chá verde, vinho tinto, cerveja, suco de uva, suco de limão, suco natural, sucos à base de soja, } \\
\text { bebida de soja, leite, bebidas fermentadas, água, café }\end{array}$ \\
Cereais & Aveia, linhaça, farelo de trigo, barra de cereal, pão integral ou similar, granola, arroz integral \\
Hortaliças & Acelga, alho, cebola, couve de bruxelas, couve flor, couve, brócolis, beringela, repolho, rúcula, \\
& tomate, milho verde, alface, agrião, cenoura crua \\
Óleos e Temperos & Azeite de oliva, óleo de soja, óleo de girassol, óleo de milho, óleo de canola, manteiga, margarina \\
Leguminosas & Proteína texturizada de soja, soja em grão, ervilha, lentilha, feijão \\
Frutas & Abacaxi, acerola, banana,caju, caqui, melão, morango, pêra, uva vermelha, goiaba, laranja, maçã, \\
& mamão, manga, melancia, pêssego, pitanga, tangerina \\
\hline
\end{tabular}


a) grupo formado pelos indivíduos que atingem pelo menos um dos critérios da recomendação para a frequência (cinco dias por semana) ou duração (150 minutos por semana).

b) grupo composto por indivíduos que não atendem nenhum dos critérios recomendados.

Ativo: aquele que cumpre as recomendações de atividades vigorosas (maior ou igual a três dias por semana e maior ou igual a 20 minutos por sessão) e/ou atividade moderada ou caminhada (maior ou igual a cinco dias por semana e pelo menos 30 minutos por sessão) e/ou qualquer atividade somada maior ou igual a cinco dias por semana e pelo menos 150 minutos por semana.

Muito ativo: aquele indivíduo que cumpre as recomendações de atividades vigorosas (maior ou igual a cinco dias por semana e 30 minutos por sessão) ou atividades vigorosas (pelo menos três dias por semana e com 20 minutos por sessão acrescidos de atividade moderada ou caminhada em pelo menos cinco dias por semana e $30 \mathrm{mi}$ nutos por sessão).

O Estado Nutricional (EN) foi avaliado pelo Índice de Massa Corporal (IMC) de acordo com a altura e o peso autorreferidos pelos participantes, por meio da equação IMC= peso $(\mathrm{kg}) /$ altura $(\mathrm{m})^{2}$, de acordo com os critérios propostos pela OMS, 2005, elaborados para indivíduos adultos de ambos os sexos. Esse critério classifica em baixo peso (IMC $\leq 18,5)$, faixa recomendável $(18,5 \leq I M C \leq 24,9)$, sobrepeso $(25 \leq I M C \leq 29,9)$, obesidade । $(30 \leq \mathrm{IMC} \leq 34,9)$, obesidade II $(35 \leq \mathrm{IMC} \leq 39,9)$ e obesidade III (IMC $\geq 40)$.

\section{Coleta de dados}

O convite para aderir à pesquisa e à coleta de dados foi enviado para toda a população-alvo através de e-mail com o link para acesso aos questionários, à política de privacidade e de segurança do site, às informações sobre a metodologia adotada na pesquisa, ao esclarecimento de dúvidas, ao Termo de Consentimento Livre e Esclarecido, além de acesso às orientações do Comitê de Ética em Pesquisas e aos pesquisadores. Protocolo apro- vado pelo Comitê de Ética e Pesquisa da Universidade Estadual de Campinas, Protocolo n 143/2004.

Autenticação digital com uso de senha foi adotada para assegurar que o usuário preenchesse os questionários apenas uma vez. Não houve qualquer tipo de identificação pública dos participantes.

\section{Análises estatísticas}

As análises de variabilidade da dieta de acordo com os alimentos presentes na pesquisa foram elaboradas por meio da utilização do software Dietsys. Foi realizada análise descritiva e da normalidade dos dados, bem como teste $t$ para comparação de médias em dados paramétricos (IMC e NAF). Foi utilizada a análise de correlação de Spearman, em dados não paramétricos, com o objetivo de encontrar associações entre as variáveis relacionadas ao consumo de alimentos. O software utilizado foi o Statistical Package for the Social Sciences 15.0. (SPSS)

\section{RES U LTA DOS}

Na amostra, formada por 130 homens $(42,0 \%)$ e 173 mulheres $(57,1 \%), 72,3 \%$ das mulheres apresentaram estado nutricional na faixa de normalidade, e $16,8 \%$, sobrepeso; $47,7 \%$ dos homens apresentaram sobrepeso, e 47,0\% estavam na faixa de normalidade. Casos de obesidade foram observados em ambos os sexos, no entanto apenas os homens apresentaram obesidade grau III (obesidade mórbida) (Tabela 1).

Quanto às características gerais da amostra, as mulheres mostraram valores médios menores quanto à idade e ao IMC, e valores absolutos maiores entre os não tabagistas. Elas são mais ativas em atividade física (63,0\%), menos sedentárias, além de mais prevalentes nos cursos de graduação, pós-graduação e entre os servidores não docentes. A análise do NAF mostrou valores muito próximos das mulheres ativas e muito ativas (72,9\%) em relação aos homens, que também reportaram valores altos nessas condições soma- 
712 ES MACIEL et al.

Tabela 1. Médias, Desvios-Padrão e Frequências das características gerais dos voluntários da comunidade do Campus "Luiz de Queiroz", classificados por sexo. Piracicaba (SP), 2006.

\begin{tabular}{|c|c|c|c|c|c|}
\hline \multirow{2}{*}{$\begin{array}{l}\text { Variáveis } \\
\text { Idade (anos) }\end{array}$} & & \multicolumn{2}{|c|}{ Homens $n=130$} & \multicolumn{2}{|c|}{ Mulheres $n=173$} \\
\hline & & \multicolumn{2}{|c|}{$M=35,09, D P=12,16$} & \multicolumn{2}{|c|}{$M=31,23, D P=10,89$} \\
\hline \multirow[t]{4}{*}{$\mathrm{IMC}\left(\mathrm{kg} / \mathrm{m}^{2}\right)$} & Mínimo & 18,12 & & 15,92 & \\
\hline & Máximo & 40,82 & & 39,97 & \\
\hline & Média & 25,90 & & 22,80 & \\
\hline & & $n$ & $\%$ & $n$ & $\%$ \\
\hline \multirow[t]{2}{*}{ Tabagismo } & Sim & 8 & 6,2 & 18 & 10,4 \\
\hline & Não & 122 & 93,8 & 155 & 89,6 \\
\hline \multirow[t]{4}{*}{ NAF } & Muito ativo & 24 & 18,4 & 18 & 9,9 \\
\hline & Ativo & 68 & 52,3 & 114 & 63,0 \\
\hline & Insuficiente ativo & 21 & 16,2 & 25 & 13,8 \\
\hline & Sedentário & 17 & 13,1 & 16 & 8,8 \\
\hline \multirow[t]{4}{*}{ Categoria } & Aluno graduação & 35 & 26,9 & 56 & 32,4 \\
\hline & Aluno pós-graduação & 30 & 23,1 & 57 & 32,9 \\
\hline & Servidor docente & 40 & 30,8 & 11 & 6,4 \\
\hline & Servidor não docente & 25 & 19,2 & 49 & 28,3 \\
\hline $\mathrm{N}$ & & 130 & 100,0 & 173 & 100,0 \\
\hline
\end{tabular}

IMC: Índice de Massa Corporal; NAF: Nível de Atividade Física; M: Média; DP: Desvio-Padrão.

das de atividade física (70,7\%). A análise estatística mostrou diferenças significantes $(p \leq 0,001)$ entre os sexos, com as mulheres mais frequentes entre os tabagistas, além de IMC mais adequado aos valores recomendados para a faixa de normalidade.

Os resultados de variabilidade alimentar, de acordo com os alimentos listados no QFA, são apresentados na Tabela 2 .

A análise de correlação indicou nível de significância entre os grupos alimentares, entretanto os resultados não mostraram forte correlação entre eles (Tabela 3) tanto para o sexo feminino quanto para o masculino. A frequência na ingestão de cereais e leguminosas apresentou valores que não alcançam uma porção diária, sendo também observados baixos valores para o grupo das frutas, segundo recomendação da OMS.

A maior correlação no grupo feminino foi notada entre as variáveis que são fontes de fibras (verduras e cereais) e as bebidas, enquanto para o sexo masculino a maior correlação ocorreu entre o grupo de óleos e temperos e as verduras e leguminosas.
A menor correlação para as mulheres ocorre entre o grupo de leguminosas e bebidas, e para os homens, entre os cereais e as bebidas. Não houve correlação significativa entre grupos de alimentos, IMC ou NAF em ambos os sexos.

\section{DISCUSS Ã O}

Este estudo mostra a inadequação dos hábitos alimentares associada à prevalência de sobrepeso e obesidade em comunidade universitária, fato que estimula a discussão de estratégias de promoção da saúde, estruturadas por ações dirigidas a essa comunidade e que priorize a orientação nutricional e o estímulo da prática do exercício físico.

As prevalências de sobrepeso e obesidade, diferenciadas entre homens e mulheres e observadas neste estudo, permitem caracterizar estados nutricionais bem distintos entre os sexos, possibilitando ampliar a discussão das relações de causa e consequência entre os determinantes dessas variações nas comunidades universitárias. Das pessoas investigadas, $16,8 \%$ das mulheres e $47,7 \%$ dos homens apresentaram sobrepeso. 
Tabela 2. Variáveis do consumo alimentar (porções e frequência) da comunidade do Campus "Luiz de Queiroz". Piracicaba (SP), 2006.

\begin{tabular}{|c|c|c|c|c|}
\hline \multirow{2}{*}{ Grupos } & \multirow{2}{*}{ Média } & \multirow{2}{*}{ Desvio-Padrão } & \multicolumn{2}{|c|}{ Valores em grama } \\
\hline & & & Mínimo & Máximo \\
\hline \multicolumn{5}{|l|}{ Bebidas } \\
\hline Número de porções/dia & 4,60 & 1,51 & 1,20 & 11,10 \\
\hline Quantidade em gramas/dia & 899,38 & 286,76 & 157,70 & 2204,70 \\
\hline Frequência de consumo semanal & 2,62 & 1,00 & 1,00 & 7,00 \\
\hline Frequência de consumo mensal & 6,34 & 1,86 & 1,00 & 12,00 \\
\hline \multicolumn{5}{|l|}{ Óleos e temperos } \\
\hline Número de porções/dia & 1,95 & 1,40 & 0 & 7,00 \\
\hline Quantidade em gramas/dia & 17,04 & 12,19 & 0 & 60,00 \\
\hline Frequência de consumo semanal & 1,47 & 1,12 & 0 & 7,00 \\
\hline Frequência de consumo mensal & 3,19 & 1,32 & 0 & 7,00 \\
\hline \multicolumn{5}{|l|}{ Leguminosas } \\
\hline Número de porções/dia & 0,79 & 0,72 & 0 & 3,10 \\
\hline Quantidade em gramas/dia & 63,68 & 59,98 & 0 & 220,00 \\
\hline Frequência de consumo semanal & 0,53 & 0,54 & 0 & 3,00 \\
\hline Frequência de consumo mensal & 1,97 & 1,18 & 0 & 5,00 \\
\hline \multicolumn{5}{|l|}{ Hortaliças } \\
\hline Número de porções/dia & 2,84 & 2,14 & 0,10 & 11,40 \\
\hline Quantidade em gramas/dia & 90,44 & 74,39 & 3,10 & 381,50 \\
\hline Frequência de consumo semanal & 1,83 & 1,59 & 0 & 8,00 \\
\hline Frequência de consumo mensal & 9,62 & 3,28 & 1,00 & 15,00 \\
\hline \multicolumn{5}{|l|}{ Cereais } \\
\hline Número de porções/dia & 0,86 & 1,18 & 0 & 7,00 \\
\hline Quantidade em gramas/dia & 37,55 & 53,31 & 0 & 270,00 \\
\hline Frequência de consumo semanal & 0,60 & 0,91 & 0 & 4,00 \\
\hline Frequência de consumo mensal & 2,08 & 1,77 & 0 & 7,00 \\
\hline \multicolumn{5}{|l|}{ Frutas } \\
\hline Número de porções/dia & 1,24 & 1,35 & 0 & 8,30 \\
\hline Quantidade em gramas/dia & 147,16 & 160,43 & 0 & 106,23 \\
\hline Frequência de consumo semanal & 0,71 & 1,12 & 0 & 6,00 \\
\hline Frequência de consumo mensal & 5,50 & 2,60 & 0 & 13,00 \\
\hline
\end{tabular}

Tabela 3. Correlações significantes entre as variáveis do consumo alimentar da amostra de voluntários da comunidade do Campus "Luiz de Queiroz", de acordo com o sexo. Piracicaba (SP), 2006.

\begin{tabular}{|c|c|c|c|c|c|c|}
\hline \multirow{2}{*}{ Variáveis } & \multicolumn{6}{|c|}{ Sexo } \\
\hline & r & $p$ & $n$ & $r$ & $p$ & $n$ \\
\hline Leguminosas X Bebidas & 0,273 & $0,000^{* *}$ & 173 & 0,241 & $0,006^{\star *}$ & 130 \\
\hline Hortaliças X Bebidas & 0,482 & $0,000^{* *}$ & 173 & 0,363 & $0,000^{\star *}$ & 130 \\
\hline Cereais $X$ Bebidas & 0,452 & $0,000^{* *}$ & 173 & 0,195 & $0,026^{*}$ & 130 \\
\hline Leguminosas X Hortaliças & 0,375 & $0,000^{* *}$ & 173 & 0,382 & $0,000^{* *}$ & 130 \\
\hline Cereais X Leguminosas & 0,310 & $0,000^{* *}$ & 173 & 0,286 & $0,001^{* *}$ & 130 \\
\hline Cereais X Hortaliças & 0,325 & $0,000^{* *}$ & 173 & 0,330 & $0,000^{* *}$ & 130 \\
\hline
\end{tabular}

${ }^{*}$ Correlação significante ao nível $p \leq 0,05 ;{ }^{* *}$ Correlação significante ao nivel de $p \leq 0,001$. 
Dentre os muitos determinantes possivelmente relacionáveis ao sobrepeso e à obesidade, há que se considerar, nesta amostra, o nível educacional que, apesar de não ter sido avaliado, pode ser considerado elevado pelo perfil das exigências para estabelecimento de vínculo com a universidade, tanto para os acadêmicos (vestibulares) como para os servidores (processos seletivos). Outros determinantes referem-se às possíveis diferenças de faixa etária entre os sexos, que, neste estudo, não foram observadas e, finalmente, à não verificação da composição corporal, que poderia indicar com maior exatidão o sobrepeso para o sexo masculino, já que o IMC, quando utilizado para homens, pode sofrer acréscimo no valor pelo fato de o sexo masculino possuir maior massa muscular do que o feminino. Outros dois importantes determinantes, que serão discutidos posteriormente neste artigo, são capazes de influenciar fortemente o estado nutricional: o primeiro está relacionado ao consumo alimentar bastante dependente dos hábitos alimentares; o segundo, vinculado à adoção de estilo de vida sedentário ou com diferentes níveis de atividade física.

Estudos sobre o estado nutricional de comunidades universitárias mostraram índices de sobrepeso e de obesidade muito variados, indicando que a cultura local pode ser um fator determinante e expressivo a se considerar nos estudos sobre a adoção dos hábitos de vidaª,22-27. Esses estudos mostraram também que há uma tendência do IMC ser maior nos homens do que nas mulheres, o que sugere maiores prevalências de sobrepeso e obesidade, ponderando-se o fato de que as medidas do IMC podem mascarar condições diferenciadas da presença de massa magra elevada, comum nos homens praticantes de modalidades esportivas específicas, como levantamento de peso, lutas e musculação. Dados nacionais da Pesquisa de Orçamentos Familiares 2008-200928 indicam que, no Brasil, o excesso de peso em homens adultos é maior $(50,1 \%)$ do que nas mulheres $(48,0 \%)$ e mais prevalente nos homens com maior renda $(61,8 \%)^{28}$.
O consumo de frutas e verduras observado neste estudo foi menor que os $400 \mathrm{~g} / \mathrm{dia}$ preconizados pela OMS, e a ingestão de leguminosas e cereais, descrita pela amostra, também não atendeu à recomendação de consumo de seis porções de cereais e uma porção de leguminosas (feijões) por dia. Esses achados corroboram o estudo que identificou a inadequação de hábitos alimentares para a maioria dos estudantes da área da saúde $(79,9 \%)$, que apresentam baixo percentual de consumo para o grupo de leite e derivados $(23,0 \%)$, frutas e vegetais ( $24,9 \%$ ) e carboidratos complexos (25,9\%), e alto percentual de consumo de refrigerantes e doces $(74,0 \%)^{22}$.

Os hábitos alimentares em comunidade universitária também têm sido destacados em outros estudos, sendo observada baixa prevalência de alimentação saudável, associada à elevada ingestão de alimentos doces e gordurosos e baixa ingestão de frutas e verduras ${ }^{27,29}$. Em relação às associações entre variáveis do consumo alimentar, os resultados de outro estudo indicaram uma correlação positiva moderada $(0,30$ a 0,49) entre determinados grupos de alimentos, para ambos os sexos ${ }^{30}$. Este estudo encontrou uma associação positiva significante entre o consumo de verduras, cereais e leguminosas e a ingestão de bebidas no grupo de mulheres, o que está de acordo com as recomendações de ingestão hídrica associada ao consumo de fibras, que visa à prevenção da constipação intestinal pelo aumento do bolo fecal, diminuição da sua consistência e do tempo de trânsito intestinal ${ }^{31,32}$.

No grupo dos homens, observamos correlação significante entre o consumo de verduras e o consumo de óleos e temperos, ingredientes utilizados para realçar o sabor dos vegetais. Apesar de ter havido também na dieta dos homens correlação entre fontes de fibra e bebidas -, ela ficou abaixo da encontrada nas mulheres. De forma geral, pode-se notar que as associações são mais expressivas nas mulheres do que nos homens, indicativo de maior consumo dos alimentos listados no questionário e da existência de um maior cuidado alimentar por parte dos integrantes do sexo feminino. 
A inadequação de hábitos alimentares também foi encontrada em outros estudos realizados com comunidades universitárias, independentemente do sexo, e indica baixa frequência de consumo de frutas e vegetais ${ }^{33-35}$. O estudo realizado com grupos de universitários de León (Espanha) e Bolonha (Itália) mostrou que ambos os grupos consomem mais gordura e menos vegetais do que o recomendado, o que ocasiona alta incidência de sobrepeso e mostra hábitos diferentes da tradição mediterrânea, que é o consumo de frutas, de pescado, de cereais e de verduras. Esses resultados foram atribuídos às mudanças do padrão dietético muito comum durante o período universitário². Essa característica também pode ser a mesma dos voluntários deste estudo, já que muitos são originários de cidades do interior do estado ou até mesmo de outros estados do Brasil.

No Brasil, o consumo de frutas, verduras e legumes tem se mostrado insuficiente entre os universitários, conforme as recomendações estabelecidas para população brasileira ${ }^{33}$. Em pesquisa realizada com universitários, foi identificado que, dentre os alimentos mais rejeitados, o grupo das verduras foi o mais referido $(79,5 \%)$, seguido pelo grupo de frutas, pois $25,4 \%$ das pessoas indicaram rejeitar algum alimento desse grupo ${ }^{29}$. Hábitos alimentares adquiridos na infância e na adolescência tendem a ser reproduzidos na fase adulta e na terceira idade e estão associados a doenças crônicas não transmissíveis e condições agravantes à saúde. A universidade é espaço de produção e de transferência de conhecimento, e, dessa forma, deve ser compreendida também como espaço de promoção da saúde, com possibilidades ímpares para o desenvolvimento de ações específicas de orientação para hábitos adequados de consumo alimentar.

Quanto ao nível de atividade física, os dados demonstraram valores surpreendentemente elevados, muito próximos entre as mulheres ativas e muito ativas $(72,9 \%)$ em relação aos homens também nessa condição de atividade física $(70,7 \%)$, não havendo, no entanto, grande similaridade com os dados encontrados em outros estudos, que indicam as mulheres mais ativas que os homens ${ }^{36-39}$.

Entretanto, estudo realizado com universitários da área da saúde identificou uma realidade mais preocupante: a maioria $(65,5 \%)$ declarou-se sedentária ${ }^{22}$. O alto nível de sedentarismo também foi encontrado em estudo com universitários nos Estados Unidos da América, o que indica que $46 \%$ eram inativos ou exercitavam-se irregularmente ${ }^{40}$.

Entendemos que a relação entre sedentarismo e prática de atividade física, ou atividade no lazer ativo, é influenciada por um conjunto de determinantes bastante numerosos e de difícil normalização diante da complexidade das sociedades atuais. Exemplos de estudos nessa área mostraram prevalência de $72,5 \%$ de sedentarismo no lazer em adultos de Salvador (BA), sendo mais frequente em mulheres entre 40 e 59 anos e em homens maiores que 60 anos de idade, em pessoas com baixo nível de escolaridade e entre os casados, separados ou viúvos ${ }^{41}$. Em Portugal, os dados observados a partir de um inquérito nacional de saúde indicaram os jovens normoponderais, solteiros, não alcoolistas e adultos não tabagistas com maior probabilidade de serem fisicamente ativos; em ambos os sexos, observouse um efeito diferencial da escolaridade segundo os tipos de atividade física, sendo mulheres e homens com maior escolaridade os que apresentavam probabilidade duas vezes maior de serem fisicamente ativos no lazer do que os menos escolarizados ${ }^{42}$.

As universidades detêm uma condição estrutural e organizacional capaz de acolher ações em promoção da saúde no seu ambiente, podendo proporcionar um espaço de convívio mais saudável, que propicie a formação de indivíduos conscientes da importância da alimentação e da prática de atividade física regular para seu bem-estar ${ }^{22}$.

Os resultados deste estudo retratam uma comunidade universitária praticante de altos níveis de atividade física, mas que apresenta hábitos 
alimentares fora das recomendações orientadas para a estruturação da vida saudável, além de estado nutricional inadequado em grande parcela dos participantes da pesquisa. Esse aspecto pode ser considerado bastante grave por se tratar de adultos no início de suas vidas de formação acadêmica e de vinculação profissional produtiva.

Diante das condições semelhantes a essas aqui descritas, as universidades podem ser consideradas locais privilegiados para a implantação de estratégias de promoção da saúde, com perspectivas bastante positivas de sucesso relativo em ações de educação nutricional, visto que poucos daqueles que hoje são adultos tiveram oportunidade de acesso ao conhecimento dos hábitos alimentares saudáveis e do consumo alimentar orientado em suas etapas de formação fundamental e média, ocorridas há algumas décadas.

Os resultados do presente estudo mostram hábitos alimentares diferentes entre os sexos na comunidade universitária, sugerindo a necessidade de uma abordagem diferenciada e adequada para cada grupo.

\section{O N CLUS Ã O}

O estudo mostrou uma comunidade universitária com bom NAF, entretanto com baixo consumo alimentar de frutas, legumes e verduras, com consumo insuficiente de alimentos do grupo de cereais e leguminosas, além de estado nutricional inadequado devido à prevalência de sobrepeso e de obesidade em relação à faixa de normalidade. Esses resultados sugerem a importância na formulação e na implementação de programas de promoção da saúde dentro da universidade, com enfoque nas práticas promotoras de estilo de vida mais saudável, focadas em ações de educação nutricional para adultos, em orientação e em adequações ambientais para propiciar a prática de atividade física e de lazer ativo.

\section{COLABORADORES}

ES MACIEL contribuiu na concepção do projeto, desenvolvimento da pesquisa, coleta e análise dos dados e redação final. JG SONATI, DM MODENEZE, JS VASCONCELOS participaram da análise dos dados e redação. R VILARTA conduziu a orientação, análise dos dados, revisão e redação final.

\section{REFERÊ NCIAS}

1. Landstrom E, Hursti UKK, Magnusson M. Functional foods compensate for an unhealthy lifestyle. Some Swedish consumers' impressions and perceived need of functional foods. Appetite. 2009; 53(1): 34-43. doi: 10.1016/j.appet.2009.04.219.

2. Baldini M, Pasqui F, Bordoni A, Maranesi A. Is the Mediterranean lifestyle still a reality? Evaluation of food consumption and energy expenditure in Italian and Spanish university students. Public Health Nutr. 2008; 12(2):148-55. doi: 10.1017/S1 368980008002759.

3. Tyrovolas S, Panagiotakos D. The role of Mediterranean type of diet on the development of cancer and cardiovascular disease, in the elderly: a systematic review. Maturitas. 2010; 65(2):122-30. doi: doi:10.1016/j.maturitas.2009.07.003.

4. Frewer L, Scholderer J, Lambert N. Consumer acceptance of functional foods: issues for the future. Br Food J. 2003; 105(10):714-31. doi: 10.11 08/00070700310506263.

5. World Health Organization. Diet, nutrition and the prevention chronic diseases. Geneva: WHO; 2003. Technical Report Series, 916 [cited 2011 Jan 24]. Available from: <http://whqlibdoc.who.int/trs/ who_trs_916.pdf>

6. Brasil. Ministério da Saúde. Secretaria de Atenção à Saúde. Guia alimentar para a população brasileira: promovendo a alimentação saudável. Brasília; 2005. Série A: normas e manuais técnicos [acesso 2011 jan 28]. Disponível em: <http://bvsms.saude. gov.br/bvs/publicacoes/guia_alimentar_popula cao_brasileira.pdf>.

7. World Health Organization. The world report 2002: reducing risks, promoting healthy life. Geneva: WHO; 2002. [cited 2011 Jan 28]. Available from: <http://www.who.int/whr/2002/en/whr02_ en.pdf>.

8. Toral N, Slater B, Cintra IP, Fisberg M. Comportamento alimentar de adolescentes em relação ao consumo de frutas e verduras. Rev Nutr. 2006; 19(3):331-40. doi: 10.1590/S1415-5273200600 0300004

9. Jaime PC, Machado FMS, Westphal MF, Monteiro CA. Educação nutricional e consumo de frutas e hortaliças: ensaio comunitário controlado. Rev 
Saúde Pública. 2007; 41(1):154-57. doi: 10.1590/ S0034-89102006005000014.

10. Brasil. Ministério da Saúde. Secretaria de Vigilância em Saúde. Vigitel Brasil 2008: vigilância de fatores de risco e proteção para doenças crônicas por inquérito telefônico. Brasília: MS; 2009. Série G. Estatística e Informação em Saúde.

11. Levy RB, Castro IRR, Cardoso LO, Tavares LF, Sardinha LMV, Gomes FS, et al. Consumo e comportamento alimentar entre adolescentes brasileiros: Pesquisa Nacional de Saúde do Escolar, 2009. Ciênc Saúde Coletiva. 2010; 15(Supl. 2):3085-97. doi: 10.1590/S1413-81232010000800013.

12. Heymsfield SB, Thomas D, Nguyen AM, Peng JZ, Martin C, Shen W, et al. Voluntary weight loss: systematic review of early phase body composition changes. Obes Rev. 2011; 12(5):348-61. doi: 10.1111/j.1467-789 X.2010.00767.x.

13. Layman DK, Evans E, Baum Jl, Seyler J, Erickson DJ, Boileau RA. Dietary protein and exercise have additive effects on body composition during weight loss in adult women. J Nutr [Internet]. 2005 [cited 2011 Jan 28]; 135(8):1903-10. Available from: $<$ http://jn.nutrition.org/content/135/8/1903.full. pdf+html>.

14. Thompson FE, Byers T. Dietary assessment resource manual. J Nutr [Internet]. 1994 [cited 2011 Jan 28]; 124(11 Suppl):2245S-317S. Available from: <http:// jn.nutrition.org/content/124/11_Suppl/224 5s.full.pdf>.

15. Sousa MP, Matos MEO, Matos FJA, Machado MIL, Craveiro AA. Constituintes químicos ativos e propriedades biológicas de plantas medicinais brasileira. $2^{\text {a }}$ ed. Fortaleza: UFC; 2004.

16. Morais SM, Cavalcanti ESB, Costa SMO, Aguiar LA. Ação antioxidante de chás e condimentos de grande consumo no Brasil. R Bras Farmacogno. 2009; 19(1B):315-20. doi: 10.1590/S0102-695X200900 0200023.

17. Ferrari CK, Torres EAFS. Alimentos funcionais: quando a boa alimentação melhora a nossa saúde. Rev Port Saúde [Internet]. 2002 [acesso 2011 mar 20]; 20(2):31-4. Disponível em: <http://srec.azores. gov.pt/dre/sd/115132020201/ESA/pdfs/alimentos_ funcionais-conceito.pdf>.

18. Mader U, Martin, BW, Schutz Y, Marti B. Validity of four short physical activity questionnaires in middle-aged persons. Med Sci Sports Exerc. 2006; 38(7):1255-66. doi: 10.1249/01.mss.00002273 10.18902.28.

19. Matsudo, SM, Matsudo VR, Araújo T, Andrade D, Andrade E, Oliveira L, et al. Nível de atividade física na população do Estado de São Paulo: análise de acordo com o gênero, idade e nível socioeco- nômico, distribuição geográfica e de conhecimento. Rev Bra Ciênc Mov [Internet]. 2002 [acesso 2011 mar 20]; 10(4):41-50. Disponível em: <http:// portalrevistas.ucb.br/index.php/RBCM/article/ viewFile/469/495>.

20. Craig $C L$, Marshall $A L$, Sjöström $M$, Bauman $A E$, Booth $\mathrm{ML}$, Ainsworth $\mathrm{BE}$, et al. International physical activity questionnaire: 12-country reliability and validity. Med Sci Sports Exerc. 2003; 35(8): 1381-95. doi: 10.1249/01.MSS.0000078924.61 453.FB

21. Nahas MV. Atividade física, saúde e qualidade de vida: conceitos e sugestões para um estilo de vida ativo. Londrina: Midiograf; 2001.

22. Marcondelli P, Costa THM, Schmitz BAS. Nível de atividade física e hábitos alimentares de universitários do $3^{\circ}$ ao $5^{\circ}$ semestres da área da saúde. Rev Nutr. 2008; 21(1):39-47. doi: 10.1590/\$1415-5 2732008000100005.

23. Madureira AS, Corseuil HX, Pelegrino A, Petroski E. Associacão entre estágios de mudança de comportamento relacionados a atividade física e estado nutricional em universitários. Cad Saúde Pública. 2009; 25(10):2139-46. doi: 10.1590/S010 2-311X2009001000005.

24. Duran AS, Castillo AM, Vio Del FR. Diferencias en la calidad de vida de estudiantes universitarios de diferente año de ingresso del campus Antumapu. Rev Chilena Nutr. 2009; 36(3):200-9. doi: 10.4067/ S0717-75182009000300002.

25. Salvo VMMA. Estado nutricional e hábitos alimentares de universitários do curso de nutrição. Rev Bra Ciênc Saúde [Internet]. 2005 [acesso 2011 mar 20]; 3(5):11-5. Disponível em: <http://seer. uscs.edu.br/index.php/revista_ciencias_saude/ article/view/639/487>.

26. Arroyo I, Rocandio P, Ansotegui AL, Pascual AE, Salces BI, Rebato OE. Diet quality, overweight and obesity in university students. Nutr Hosp [Internet]. 2006 [cited 2011 Mar 20]; 21(6):673-9. Available from: <http://www.ncbi.nlm.nih.gov/pubmed/ 17147065>.

27. Petribú MMV, Cabral PC, Arruda IKG. Estado nutricional, consumo alimentar e risco cardiovascular: um estudo em universitários. Rev Nutr. 2009; 22(6):837-46. doi: 10.1590/S1415-52732009000 600005.

28. Instituto Brasileiro de Geografia e Estatística. Pesquisa de orçamentos familiares 2008-2009. Rio de Janeiro: IBGE; 2010 [acesso 2011 jul 20]. Disponível em: <http://www.ibge.gov.br/home/estatistica/ populacao/condicaodevida/pof/2008_2009/ POFpublicacao.pdf>. 
29. Vieira VCR, Priore SE, Ribeiro SMR, Franceschini SCC, Almeida LP. Perfil socioeconômico, nutricional e de saúde de adolescentes recém-ingressos em uma universidade pública brasileira. Rev Nutr. 2002; 15(3):273-82. doi: 10.1590/S1415-527320020003 00003.

30. Levin J, Fox JA. Estatística aplicada a ciências humanas. 9a ed. São Paulo: Pearson; 2004.

31. Gorman MA, Bowman C. Position of the American Dietetic Association: health implications of dietary fiber. J Am Diet Assoc. 1993; 93(12):1446-7. doi: 10.1016/j.jada.2008.08.007.

32. Marlett JA, Slavin JL. Position of the American Dietetic Association: health implications of dietary fiber. J Am Diet Assoc [Internet]. 1997 [cited 2011 Mar 20]; 97(10):1157-9. Available from: <http:// www.ncbi.nlm.nih.gov/pubmed/12146567>.

33. Feitosa EPS, Dantas CAO, Andrade-Wartha ERS, Marcellini PS, Mendes-netto RS. Hábitos alimentares de estudantes de uma universidade pública no nordeste do Brasil. Alim Nutr [Internet]. 2010 [acesso 2011 mar 20]; 21(2):225-30. Disponível em: <http://serv-bib.fcfar.unesp.br/seer/index.php/ alimentos/article/viewFile/1185/a8v21n2.pdf>.

34. Oliveras López M J, Nieto Guindo P, Agudo Aponte E, Martínez Martínez F, López García de la Serrana H, López Martínez M C. Evaluación nutricional de una población universitaria. Rev Nutr Hosp [Internet]. 2006 [acesso 2011 mar 20]; 21(2):179-83. Disponível em: <http://scielo.isciii.es/scielo.php? script=sci_arttext\&pid=S0212-161120060002 $00008 \& \operatorname{lng}=e s>$.

35. Martins Bion F, Castro Chagas MH, Santana Muniz G, Oliveira de Sousa LG. Estado nutricional, medidas antropométricas, nivel socioeconómico y actividad física en universitarios brasileños. Nutr Hosp [Internet]. 2008 [acesso 2011 mar 20]; 23(3): 234-41. Disponível em: <http://scielo.isciii.es/scielo. php?script=sci_arttext $\&$ pid $=$ S0212-1611200800 0300010\&lng=pt>.
36. Monteiro CA, Conde WL, Matsudo SM, Matsudo VR, Bonsenor IM, Lotufo PA. A descriptive epidemiology of leisure-time physical activity in Brazil, 1996-1997. Rev Panam Salud Publica. 2003; 14(4):246-254. doi: 10.1590/S1020-49892003000 900005.

37. Salles-Costa R, Werneck G, Faerstein E, Lopes C. Gênero e prática de atividade física de lazer. Cad Saúde Pública. 2003; 19(4):325-33. doi: 10.1590/S0 102-311X2003000800014.

38. Lindstrom M, Isacsson SO, Merlo J. Increasing prevalence of overweight, obesity and physical inactivity: two population-based studies 1986 and 1994. Eur J Public Health [Internet]. 2003 [cited 2011 Jul 20]; 13(4):306-12. Available from: <http:// eurpub.oxfordjournals.org/content/13/4/306. full.pdf $>$.

39. Martínez-Ros MT, Tormo MJ, Perez-Flores D, Navarro C. Physical sports activity in a representative sample of the population of Region de Murcia, Spain. Gac Sanitaria. 2003; 17(1):11-9. doi: 10.15 90/S0213-91112003000100004.

40. Pinto BM, Marcus BH. A stages of change approach to understanding college students' physical activity. J Am Coll Health. 1995; 44(1):27-31. doi: 10.1080/ 07448481.1995 .9937506$.

41. Pitanga FJ, Lessa I. Prevalência e fatores associados ao sedentarismo no lazer em adultos. Cad Saúde Pública. 2005; 21(3):870-87. doi: 10.1590/S0102-3 $11 \times 2005000300021$.

42. Camões M, Lopes C. Fatores associados à atividade física na população portuguesa. Rev Saúde Pública. 2008; 42(2):208-16. doi: 10.1590/S0034-891020 08000200004 . 